\title{
Angle-, Energy- and Spin-Resolved Photoelectron Emission Using Circularly Polarized Synchrotron Radiation
}

\author{
U. Heinzmann \\ Fakultät Physik, Universität Bielefeld, D-4800 Bielefeld, F.R.G.
}

Received August 4, 1986; accepted October 8, 1986

\begin{abstract}
With the development of BESSY a light source of circularly polarized vuv radiation with sufficiently high intensity has become available to make angleand energy-resolved photoelectron spin-polarization studies with circularly polarized radiation feasible. This paper reviews the recent experimental activities performed with free atoms, physisorbed adsorbates and solid surfaces (metals as well insulator) in the past three years. The spin-polarization of photoelectrons measured under certain conditions to be almost complete studies atomic effects as auto-ionization resonances in the gas phase as well as even in the photo-emission of adsorbates. The cross comparison of photoemission data from $\mathrm{Xe}$ in its different structural phases (free atom adsorbate in different structures and on different substrates, three dimensional crystal) yields symmetry characterizations of the electronic states (assignment of quantum numbers, determination of dipole matrix elements and phase shifts; spin resolved band mapping) and shows the ability of spin-polarization spectroscopy to build a bridge between atomic and surface physics.
\end{abstract}

\section{Introduction}

The concept of polarized photoelectrons ejected by circularly polarized light has been introduced by Fano 1969 [1]. This Fano effect was first experimentally confirmed for free Cs atoms 1970 [2], for a Cs metal layer 1971 [3], for a GaAs semiconductor 1975 [4] and for free molecules 1980 [5]. Spinorbit interaction is the essential mechanism leading to a spin polarization of photoelectrons with degrees of polarization of up to $100 \%$ (optical pumping type process). Up to 1984 experimental analysis of the electron-spin polarization in photoionization and photo-emission using circularly polarized light was restricted to angle-integrated measurements without resolution of the kinetic energy of the photoelectrons ejected; that type of experiment has been performed for many free atoms, molecules and solids (for reviews see for example [6-8]). Parts of these have used circularly polarized synchrotron radiation emitted out of the plane of the synchrotron in Bonn [9].

With the availability of intense synchrotron radiation from electron-storage rings like BESSY, fluxes of up to some $10^{11}$ circularly polarized vuv photons/s are available to study the photoelectron emission simultaneously resolved with respect to the photon polarization, the photon energy, the electron emission angle, the kinetic energy of the electrons and their spin-polarization components. Because of the excellent UHV running conditions, the technique was open for studies not only with free atoms [10], but also with solid surfaces [11] and adsorbates [12]. Meanwhile the results of many publications demonstrate the ability of this technique and of the corresponding apparatus' built at BESSY, aspects of partial fields are reviewed in detail elsewhere [13-15].

It is the purpose of this paper to give a review of recent experiments under the certain aspect to make a cross com- parison of photoemission data from Xe in its different structural phases (free atom, adsorbate in different structures and on different substrates, three dimensional crystal) and to extend this to other target systems. After a brief presentation of the experimental technique and some results of angle- and spin-resolved photoelectron spectra the joint interest of atomic and surface physics is demonstrated by the report of atomic like sharp photon-induced resonance structures observed in spin-polarization spectroscopy.

Furthermore data are reported which allow the experimental determination of the symmetries (angular momentum quantum numbers) of electron bands (atomic states): the results of spin resolved band mapping for three solids and an example of a complete quantum mechanical characterization of atomic photoionization (experimental determination of all matrix elements and phase-shift differences) are also given. The paper should indicate, how angle- and spin-resolved photoelectron spectroscopy might provide a tool to build a bridge of understanding between photoionization and photoemission of nonmagnetic targets from atoms via adsorbates up to crystals.

\section{Experimental}

Most studies of photoionization with circularly polarized radiation are hampered by the fact that most atoms and molecules have their ionization threshold in the vuv, where conventional methods for producing circularly polarized radiation by use of a quarter wave plate and a prism break down (photon energy $>10 \mathrm{eV}$ ). They can be performed, however, with synchrotron radiation, which is linearly polarized when emitted in the plane of the storage ring but is elliptically polarized with a high degree of right (left) handed circular polarization when emitted above (below) the plane. This "source" of circularly polarized vuv radiation has been used in the energy range up to $35 \mathrm{eV}$ since $1978[9,16]$ in Bonn and since 1982 at BESSY in Berlin.

The synchrotron radiation of BESSY is dispersed by a specially designed $6.5 \mathrm{~m}$ N.I. UHV monochromator (Fig. 1) of the Gillieson type [17] with the electron beam in the storage ring being the virtual entrance slit. A spherical mirror and a plane holographic grating (1200 or 3600 lines $/ \mathrm{mm}$ ) form a $1: 1$ image of the tangential point in one of the two exit slits. With a slit width of $2 \mathrm{~mm}$ a bandwidth of $0.5 \mathrm{~nm}$ and $0.1 \mathrm{~nm}$ has been achieved for the 1200 grating (first diffraction order) and for the 3600 grating (second order), respectively. Apertures movable in vertical direction are used to select radiation emitted above and below the storage-ring plane, which has positive or negative helicity, respectively. The optical degrees of polarization of the synchrotron radiation have 


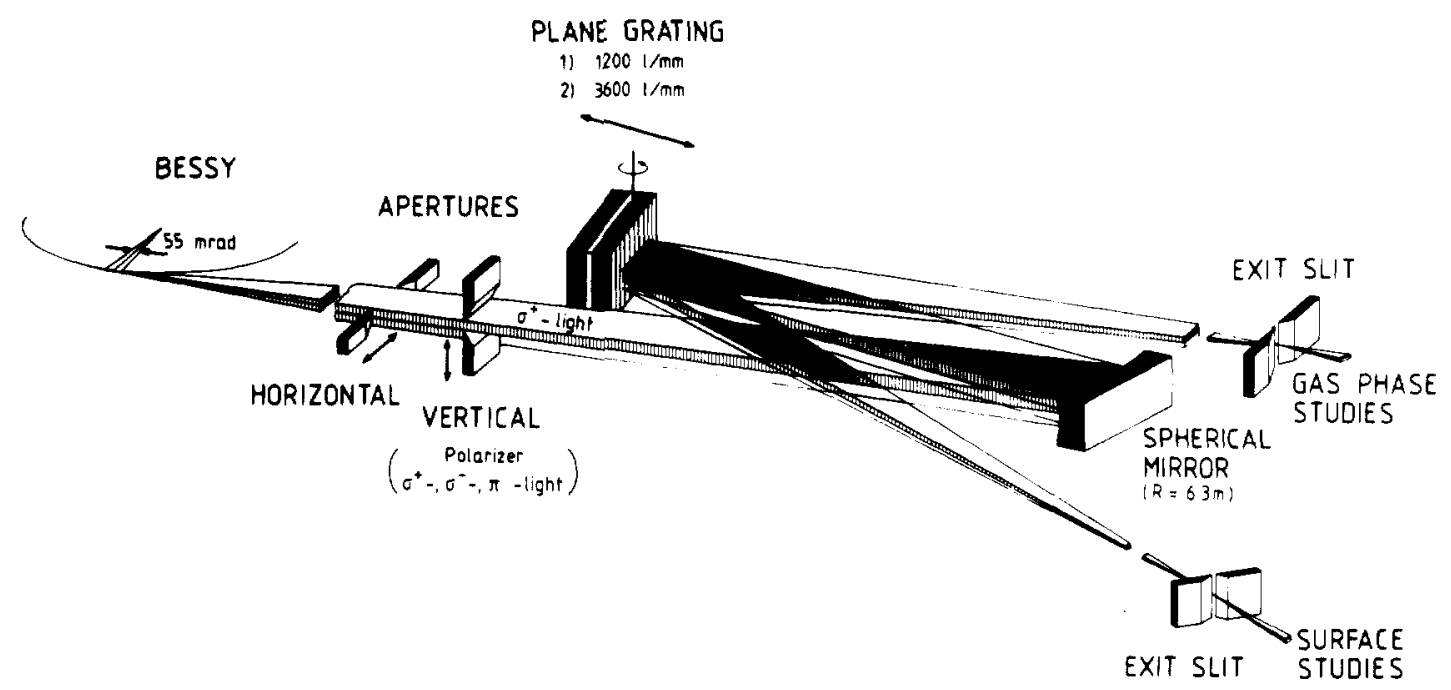

Fig. 1. Schematic diagram of the $6.5 \mathrm{~m}$ N.I. Monochromator for circularly polarized synchrotron radiation at BESSY.

been measured [10] by means of a rotatable four mirror analyzer $[9,16]$. Figure 2 upper part shows the results of the circular polarization $P_{\text {circ }}$ and the linear polarization $P_{\text {lin }}$ as functions of the vertical angle $\psi( \pm 0.1 \mathrm{mrad})$ in comparison with theoretical predictions. The lower part of Fig. 2 shows
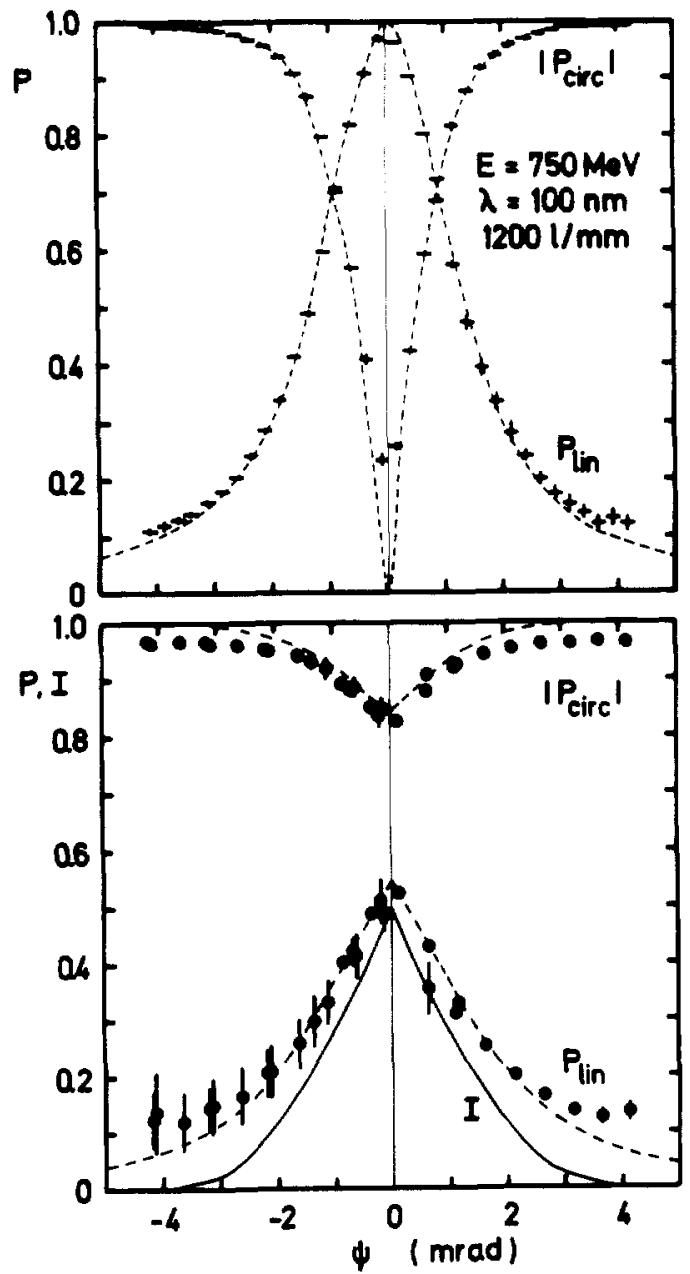

Fig. 2. Degree of circular and linear polarization, $P_{\text {in }}$ and $P_{\text {circ }}$ and intensity $I$ for synchrotron radiation with $\lambda=100 \mathrm{~nm}$ measured behind the monochromator $[10,17]$ in comparison with calculation (dashed), upper part: as function of the vertical angle $\psi( \pm 0.1 \mathrm{mrad})$, lower part: the apertures accept a vertical angular range from $\psi$ to $\pm 5 \mathrm{mrad}$. $I$ is given as fraction of the total intensity in the full vertical range from -5 to $+5 \mathrm{mrad}$. the optical data which are more relevant for practical purposes: the case when apertures are opened to get a high photon flux throughput: in the vertical acceptance range from $\psi$ above or below the storage ring plane to $+5 \mathrm{mrad}$ or $-5 \mathrm{mrad}$, respectively. Figure 2 (lower part) also shows the ratio $I$ of the intensity in this angular range to the total intensity in the full vertical acceptance. Our spin-resolved photoemission measurements are usually performed with the apertures set to accept radiation above and below $\pm 1 \mathrm{mrad}$. Thus a photon flux of up to $5 \times 10^{11}$ photons $\mathrm{s}^{-1}$ with a degree of circular polarization of $\pm 93 \%$ passes the monochromator, which is about $30 \%$ of the intensity emitted in the full vertical cone.

Behind the monochromator exit slits two apparatus are in operation, which are very similar in their set up, one for photoionization of free atoms and molecules (high vacuum system) the other for photoemission of solids and absorbates (UHV). As shown in Fig. 3 (schematic diagram of the UHV system) the elliptically polarized vuv radiation hits the phototarget under normal incidence producing photoelectrons in a region free of electric or magnetic fields. The sample is cleaned by ion bombardment, heating in oxygen and flashing in a separate preparation chamber. The crystal on top of

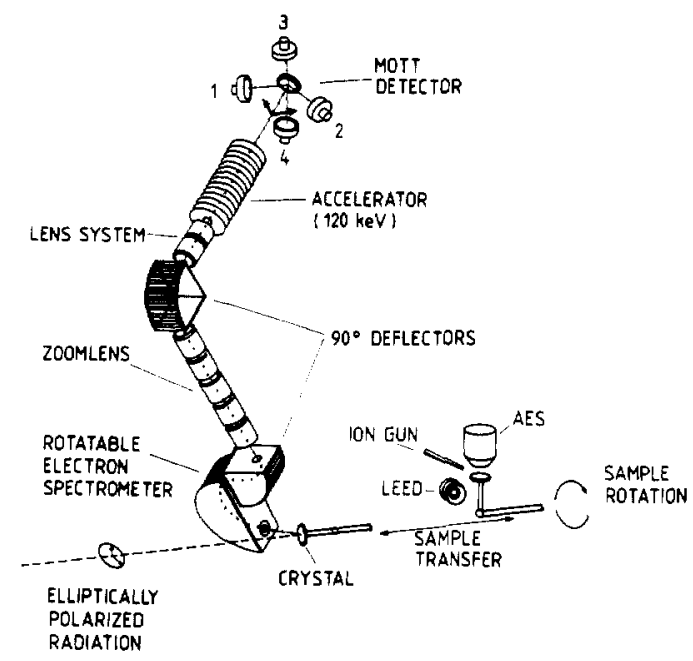

Fig. 3. Experimental set-up of the spin-, energy- and angle-resolved photoemission experiment. 
a manipulator can be cooled by use of a liquid He-cryostat to temperatures of less than $40 \mathrm{~K}$.

The photoelectrons emitted into a cone of $\pm 3^{\circ}$ are analyzed with respect to their kinetic energy by a rotatable simulated hemispherical electron spectrometer [18] and are directed by a $90^{\circ}$ electrostatic deflection along the axis of rotation of the spectrometer. This direction, which is the normal of the reaction plane, spanned by the momenta of the incoming radiation and the outgoing photoelectrons, is rotated by $45^{\circ}$ with respect to the major (and the minor) axis of the light polarization ellipse. Thus the formulae describing the angular dependences of the spin-polarization components of atomic photoelectrons become simple trigonometric relations $[19,20]$. After a second deflection the electrons are accelerated to $120 \mathrm{keV}$ and scattered at the gold foil of the Mott detector for the spin-polarization analysis. Instrumental asymmetries have been eliminated by taking advantage of the reversal of light helicity as well as by use of four additional detectors in forward scattering directions in the Mott detector (not shown in Fig. 3). Parts of the measurements performed in the apparatus at BESSY described have used another type of electron spectrometer (CMA) and spin detector (LEED) (not shown in Fig. 3) [21, 22].

\section{Angle- and spin-resolved photoelectron spectra}

When a rare gas atom is ionized by $15 \mathrm{eV}$ radiation the produced $p$ hole shows a fine structure splitting corresponding to the ionic groundstate configurations $\left({ }^{2} P_{3 / 2}\right.$ and $\left.{ }^{2} P_{1 / 2}\right)$ and resulting in two peaks in the photo-electron spectra as shown in Fig. 4 (left upper corner). The peaks are separated by the existence of the spin-orbit interaction. If they are resolved separately by means of an electron spectrometer, the photoionization measurements are sensitive to the spin-orbit coupling and, thus, all photoelectrons analyzed should be commonly spin polarized [23]. For peak 3 (in Fig. 4) of free xenon atoms ionized by $80 \mathrm{~nm}$ right handed circularly polarized vuv radiation Fig. 5 shows the angular dependences of the photoelectron intensity $I(\theta)$ as well as of the three spinpolarization components $P_{\perp}(\theta), A(\theta), P_{\mathrm{p}}(\theta)$ of the vector $\boldsymbol{P}(\theta)$ as function of the emission angle $\theta$. The curves shown in Fig. 5 are fit curves according to the experimental results

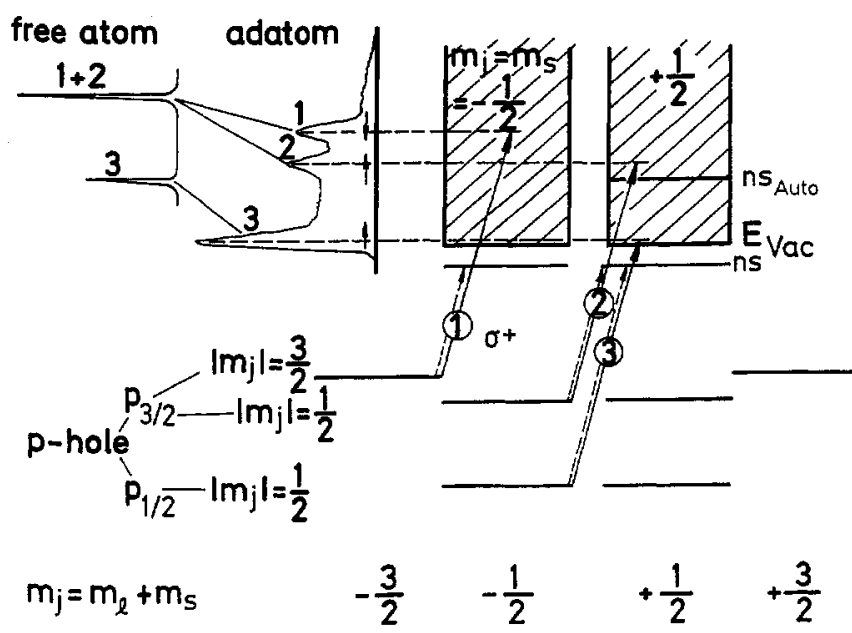

Fig. 4. Level diagram for photoionization of rare gas atoms and normal photoemission of rare gas adsorbates with circularly polarized radiation $\left(\sigma^{+}\right.$, selection rule $\Delta m_{j}=+1$ ). Arrows indicate non-vanishing transitions. The three transitions $1-3$ give rise to the three peaks in the photoelectron spectrum [12], whereas peaks 1 and 2 coincide for free atoms [24].
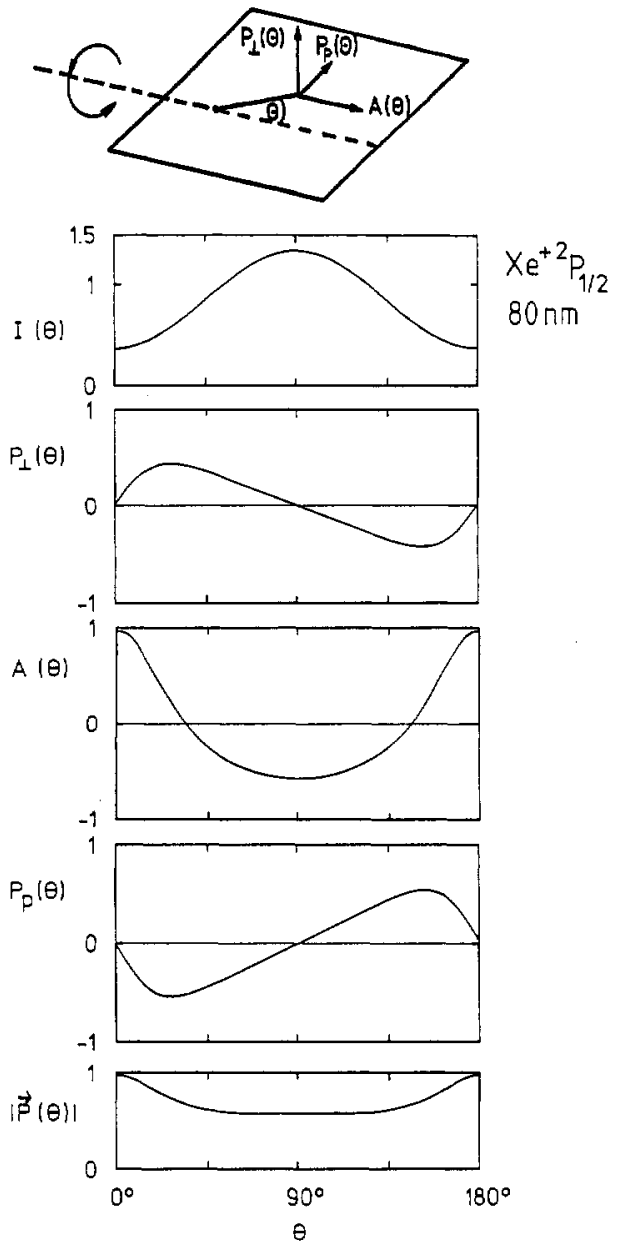

Fig. 5. Fit-curves of the experimental results $[10,24]$, describing the angular dependences of the photoelectron intensity $I(\theta)$, of the 3 components and the length of the spin-polarization vector for photoionization of xenon atoms at $80 \mathrm{~nm}$; the ion is in the ${ }^{2} P_{l, 2}$ state.

$[10,24]$. The spin-polarization component $P_{\perp}(\theta)$, perpendicular to the reaction plane (given on top of Fig. 5), has the average value zero and is thus only observed in an angle resolved photoionization experiment at $\theta$ different from $0,90^{\circ}, 180^{\circ}$ [24]; the angular dependence is given by $\xi \sin \theta \cdot \cos \theta$ modulated by the differential cross section $[\propto I(\theta)]$ with $\xi$ as corresponding spin parameter. The situation of the component $P_{\mathrm{p}}(\theta)$ also perpendicular to the direction of radiation but in the reaction plane is very similar to $P_{\perp}$ and is characterized by the spin parameter $\alpha$. Opposite to that the third spin-polarization component $A(\theta)$ parallel to the photon spin has a nonvanishing angle-averaged value $A$ (Fano effect) and is angular modulated by the second Legendre polynomial $P_{2}(\cos \theta)$ with the asymmetry parameter $\alpha$ similar to $I(\theta)$ with the well-known asymmetry parameter $\beta$.

It is worth noting that within the error limits the photoelectrons emitted into the forward or backward direction $\left(\theta=0,180^{\circ}\right)$ have been found [10] to be completely spin polarized (Fig. 5 middle part). This comes from the fact that at $\theta=0$ the continuum wavefunctions have pure $s$-character, the $d$ waves vanish there. Fig. 4 demonstrates why the spin polarization is complete: taking into account the transition selection rule for right handed circularly polarized light $\Delta m_{j}=+1$ the $p_{1 / 2}$ electron can only be photoexcited into the $m_{s}=+\frac{1}{2}$ continuum state. On the other hand transitions from the $p_{3 / 2}|m|_{j}=3 / 2$ and $|m|_{j}=1 / 2$ states create $s$ photoelectrons of opposite (but complete) spin polarization. In the 


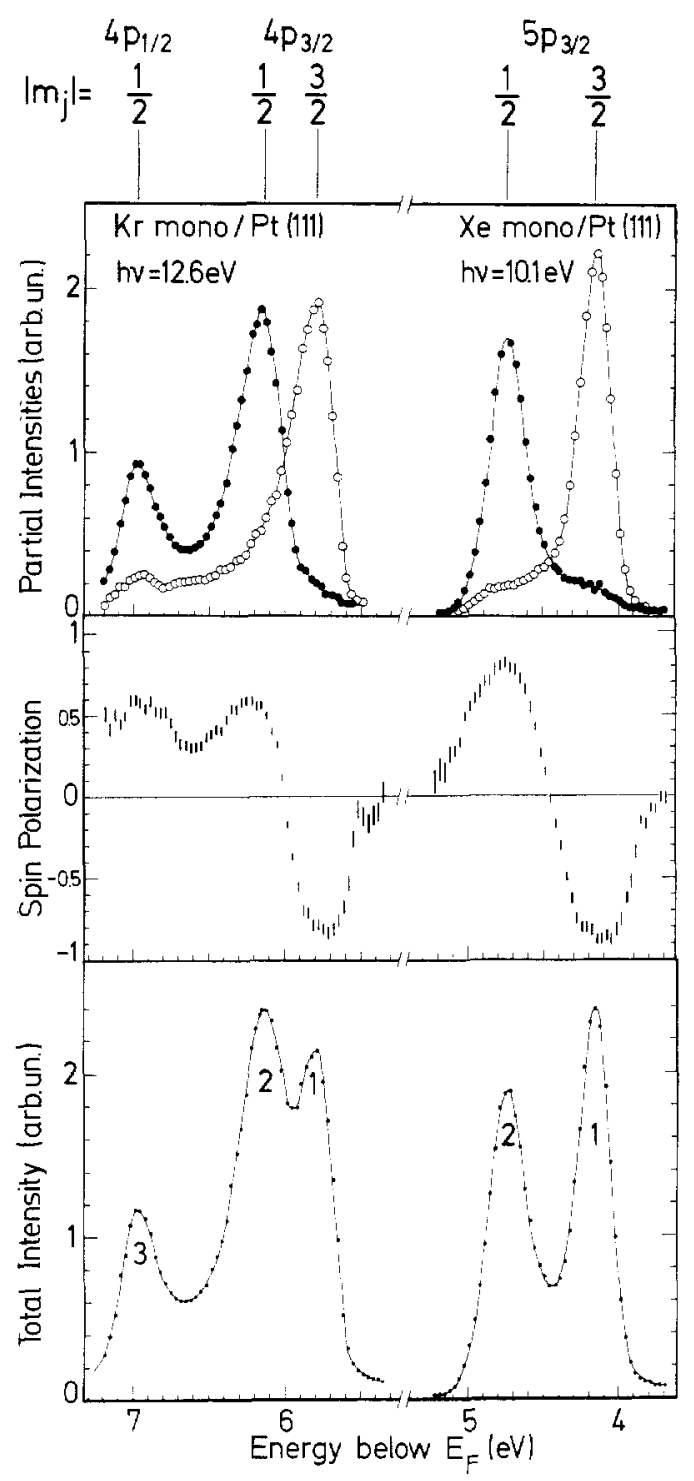

Fig. 6. Spin resolved photoelectron spectra of a monolayer $\mathrm{Kr}$ and $\mathrm{Xe}$ on Pt( 111 l). Experimental results [12] of the total (spin independent) intensity (lower part), electron spin polarization (middle part), partial intensities for spin up (full) and spin down (open) (upper part).

case of adsorbates the reduction of symmetry by the presence of the surface induces a further splitting of the energy levels as shown in Fig. 4. Measurements of the photoelectron-spin polarizations yield information about the quantum numbers of the $p$-hole states, the photoelectrons come from [12]. Figure 6 (lower part) gives the photoelectron spectra of $\mathrm{Kr}$ and $\mathrm{Xe}$ on $\mathrm{Pt}\left(\begin{array}{l}11 \\ 1\end{array}\right)$ at full monolayer coverage. Combination with the corresponding spin-polarization values measured (almost complete negative and positive polarizations) (middle part) results in the partial spectra for spin up and down drawn in the upper part of the Fig. 6. The peak structure is unambiguously correlated with either "spin up" of "spin down" with the consequence that the peaks 1, 2, 3 can be characterized by the atomic hole state quantum numbers as given on top of Fig. 6 and schematically set up in Fig. 4 . The sequence of the $m_{j}$-quantum numbers indicates that the $1-2$ splitting is caused by lateral adatom interactions or crystal field splitting but not by other mechanisms discussed in the literature as substrate-induced relaxation effects in the final ionic state or the formation of molecular orbitals $[12,15]$.

Because in photoemission of solids normal to the surface

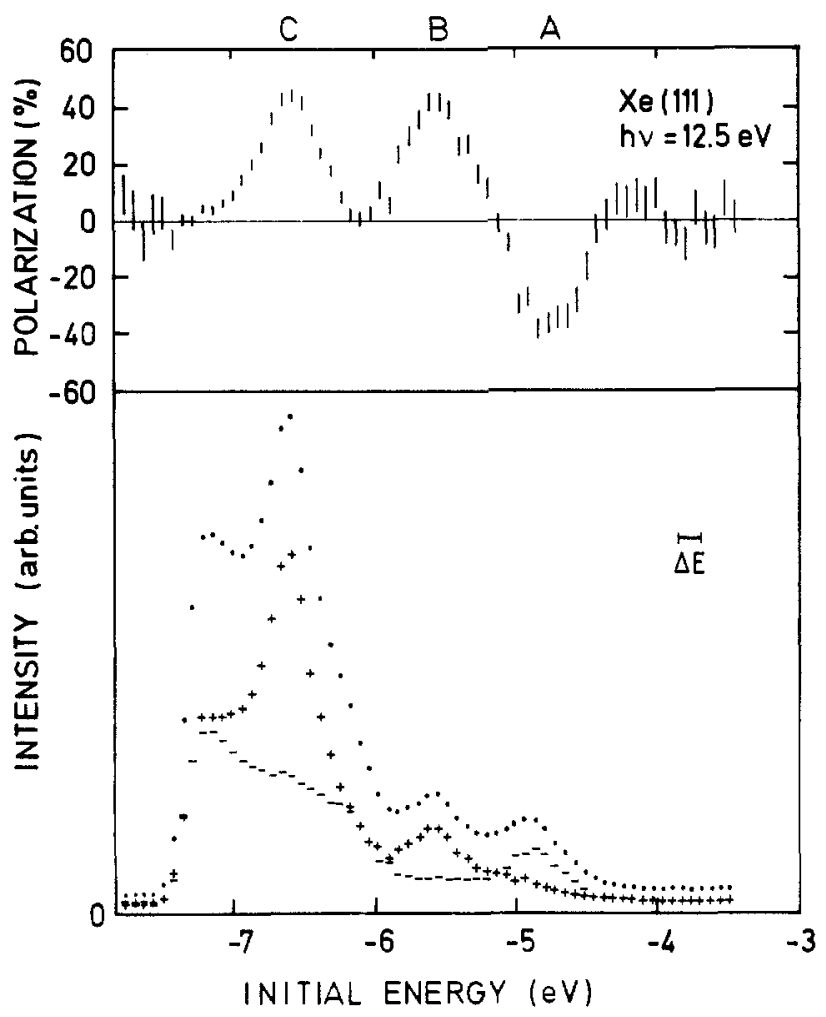

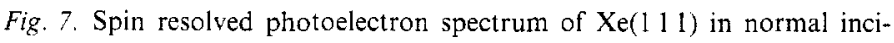
dence and normal photoemission [25]; upper part: spin polarization, lower part: total intensity $(\cdot)$, partial intensities for spin up $(+)$ and spin down $(-)$. The initial energy scale refers to the Fermi level of the substrate Pt(111).

always only $s$ partial waves escape into the vacuum, the characterization of the symmetries of the electronic bands by means of the sign of the photoelectron-spin polarization can be performed also at solids, which are more complicated than free rare gas atoms or rare gas adsorbates, in the style demonstrated in Fig. 4. Figure 7 shows the photoelectron spectra and corresponding spin polarizations [25] for the three dimensional $\mathrm{Xe}\left(\begin{array}{lll}1 & 1 & 1\end{array}\right)$ insulator crystal. The existence and sequence of the three peaks $\mathrm{A}, \mathrm{B}, \mathrm{C}$ is very similar to the monolayer regime (Peaks 1, 2, 3 in Fig. 6) resulting in the same assignment of quantum numbers as in Fig. 4 or Fig. 6 ( $p$-hole states). As shown in section 5 they correspond to electronic bands with symmetries $\Lambda_{4.5}, \Lambda_{6}$ and $\Lambda_{6}$. The lower spin-polarization values measured (upper part Fig. 7) are due to the pronounced inelastic background of electrons as seen in the intensity spectra (lower part of Fig. 7).

Figure 8 gives an example of a spin resolved photoelectron spectrum of a metal [Ir(1 111$)$ ] [26], after similar data have been published already for $\operatorname{Pt}(111)[11,21]$ two years ago. Photoemission of these metals in normal incidence and normal emission occurs due to direct interband transitions from four $d$ bands below the Fermi energy with symmetries $\Lambda_{4+5}^{3}$, $\Lambda_{6}^{3}, \Lambda_{6}^{3}, \Lambda_{4+5}^{3}$ (see Section 5). This has been experimentally verified by the photoelectron spin polarization spectroscopy [11] and is demonstrated in Fig. 8. The lower part shows the pronounced structure of four peaks in the photoelectron spectrum. The sequence of the electron spin polarization sign (middle part) has been found to be negative, positive, positive, negative corresponding to the symmetries $\Lambda_{4+5}^{3}$ or $\Lambda_{6}^{3}$ as in the case of $\mathrm{Xe}\left(\begin{array}{lll}1 & 1 & 1\end{array}\right)$. The high polarization of up to $80 \%$ exceeds all data ever previously measured for other nonmag- 


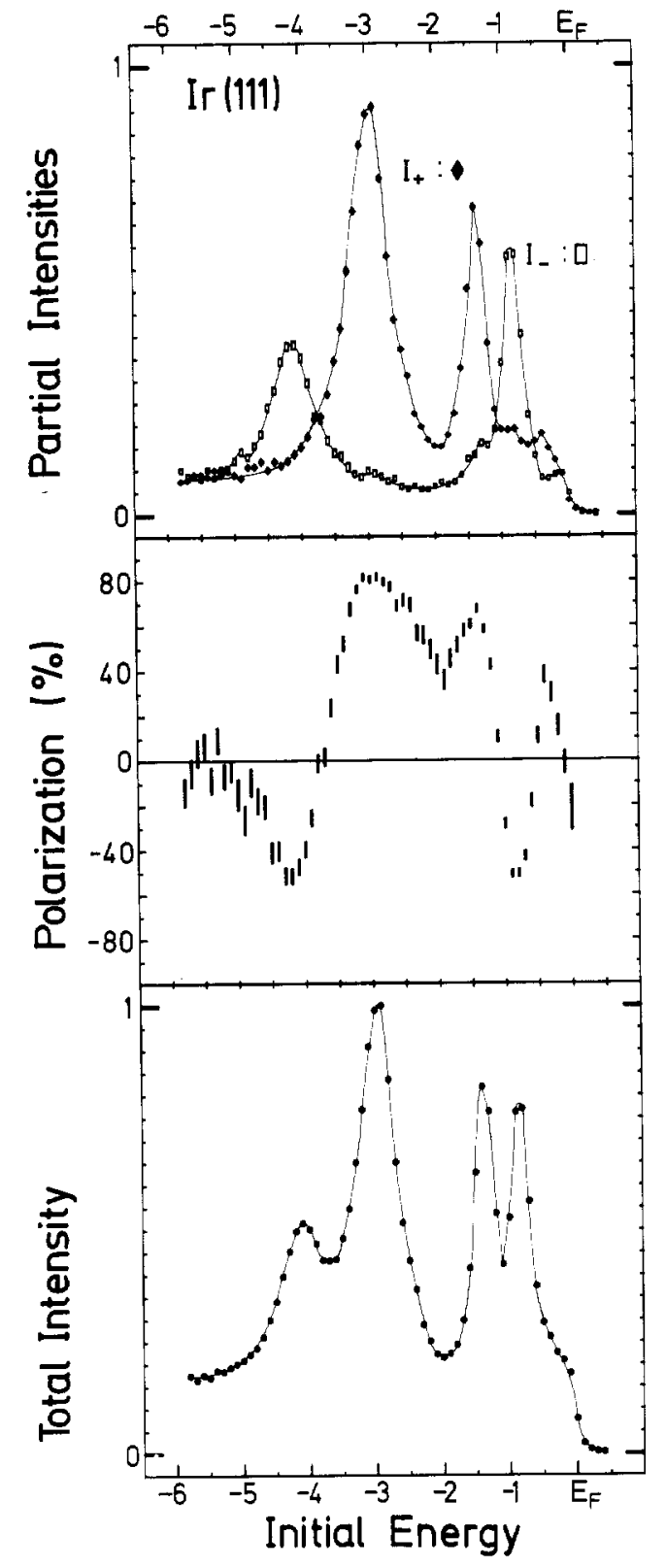

Fig. 8. Spin resolved photoelectron spectrum of $\operatorname{Ir}\left(\begin{array}{lll}1 & 1 & 1\end{array}\right)$ in normal incidence and normal photoemission at $16 \mathrm{eV}$ photon energy [26]; lower part: total (spin independent) intensity; middle part: electron spin polarization; upper part: partial intensities for spin up (full) and spin down (open).

netic three dimensional solid state systems. Combination of intensity and spin-polarization values yields again the partial photoelectron spectra with spin up and spin down (upper part) which are used for a spin resolved band mapping (see Section 5). It is worth noting that the positions of the peaks in the partial spectra are not identical with those in the total intensity spectrum (lower part) [26] which shows the necessity of performing band mapping spin resolved. The small structures close to the Fermi energy in the upper and middle part of Fig. 8 connected with the shoulder in the lower part originate from transitions into higher bands and are discussed in Section 5.

In order to avoid confusion it is emphasized that this paper uses a joint definition of the sign of electron-spin polarization. For the component parallel to the direction of the incoming light, which is the only non vanishing one for the case of normal incidence and normal photoemission, positive or negative mean that photon spin and electron spin are parallel or antiparallel, respectively. This makes sense, if photoionization of free atom and photoemission of adsorbates and crystals are compared with each other. This definition is in agreement with the literature of spin resolved photoelectron spectroscopy of free atoms, molecules, adsorbates, and the Xe insulator $[9,10,12,15,19,25]$ but unfortunately in disagreement with some publications concerning photoemission of metals $[11,21,22,26]$. It avoids any difficulties of a necessity to define a quantization axis ( $z$ axis, often chosen as normal to the solid surface) and to describe what "right" or "left" handed circularly polarized light is (there are two definitions, the optical and the helicity one).

\section{Spin resolved resonances}

In the schematic energy-level diagram of Fig. 4 the existence of excited atomic states below the photoionization threshold is indicated by the symbol $n s$ which represents some Rydberg series. These excited states can be occupied by electrons which correspond to either a $p_{1 / 2}$ or a $p_{3 / 2}$ hole (dashed arrows). In Fig. 4 which is of the "one electron picture" type a discrete excitation from $p_{1 / 2}$ to $n s$ energetically corresponds with a photoionization from $p_{3 / 2}$ to the continuum above the threshold $\left(E_{\mathrm{Vac}}\right)$; this correspondence creates an autoionization resonance in the photoelectron emission. This is indicated by the symbol $n s_{\text {Auto }}$ in Fig. 4.

Fig. 9 shows what happens if vuv radiation of the energy between 8 and $16 \mathrm{eV}$ is absorbed by free xenon atoms. There are three regions: the discrete range of pure absorption below the first photoionization threshold, the autoionization range between first and second ionization threshold corresponding to the different $p_{3 / 2}$ and $p_{1 / 2}$ hole energies (Fig. 4) and the range beyond the second threshold, where always two kinds of photoelectrons are produced, fast electrons which correspond to the ${ }^{2} P_{3 / 2}$ ionic state and slow one (to ${ }^{2} P_{1 / 2}$ ionic state). The upper part of Fig. 9 shows how the different Rydberg series [27] converging to the second limit influence the photoionization process; the full curve describes the photoelectron intensities measured [9] in comparison with a theoretical prediction [28]. The lower part of Fig. 9 shows the angle-averaged spin polarization $A$ (parallel to the photon spin, see section 3 ). The experimental results (error bars [9]) in reasonable agreement with different MQDT-theories (dashed [28], dotted [9], full [29]) demonstrate a pronounced resonance behavior of the spin polarization in the autoionization region, too.

In adsorbate systems resonance features have recently been observed below the adsorbate photoemission thresholds $[30,31]$. Figure 10 shows how the photoelectron intensities with kinetic energies between 0 and $2 \mathrm{eV}$ dramatically increase if the photon energies (below the photo-emission threshold at $9.3 \mathrm{eV}$ ) are a little bit varying between 8 and $8.2 \mathrm{eV}$ [31]. These additional electrons (dashed area in Fig. 10) are only produced if there is an adsorbate layer on the substrate (in Fig. $10 \mathrm{Xe}$ monolayer on $\operatorname{Ir}\left(\begin{array}{lll}1 & 1 & 1\end{array}\right)$ ). Figure 11 shows the results for $\mathrm{Xe}$ on $\operatorname{Pt}\left(\begin{array}{lll}1 & 1 & 1\end{array}\right)[12,30]$ and on graphite $[13,30,31]$. At photon energies below the first threshold $(9.2 \mathrm{eV}$ and $9.8 \mathrm{eV}$, respectively) resonance enhancement of the electron spectra occurs very narrow to the atomic Xe $5 p-6 s$ Rydbergstate transition (similar to Fig. 10 but total intensity as function of the light energy in Fig. 11). The resonances were 


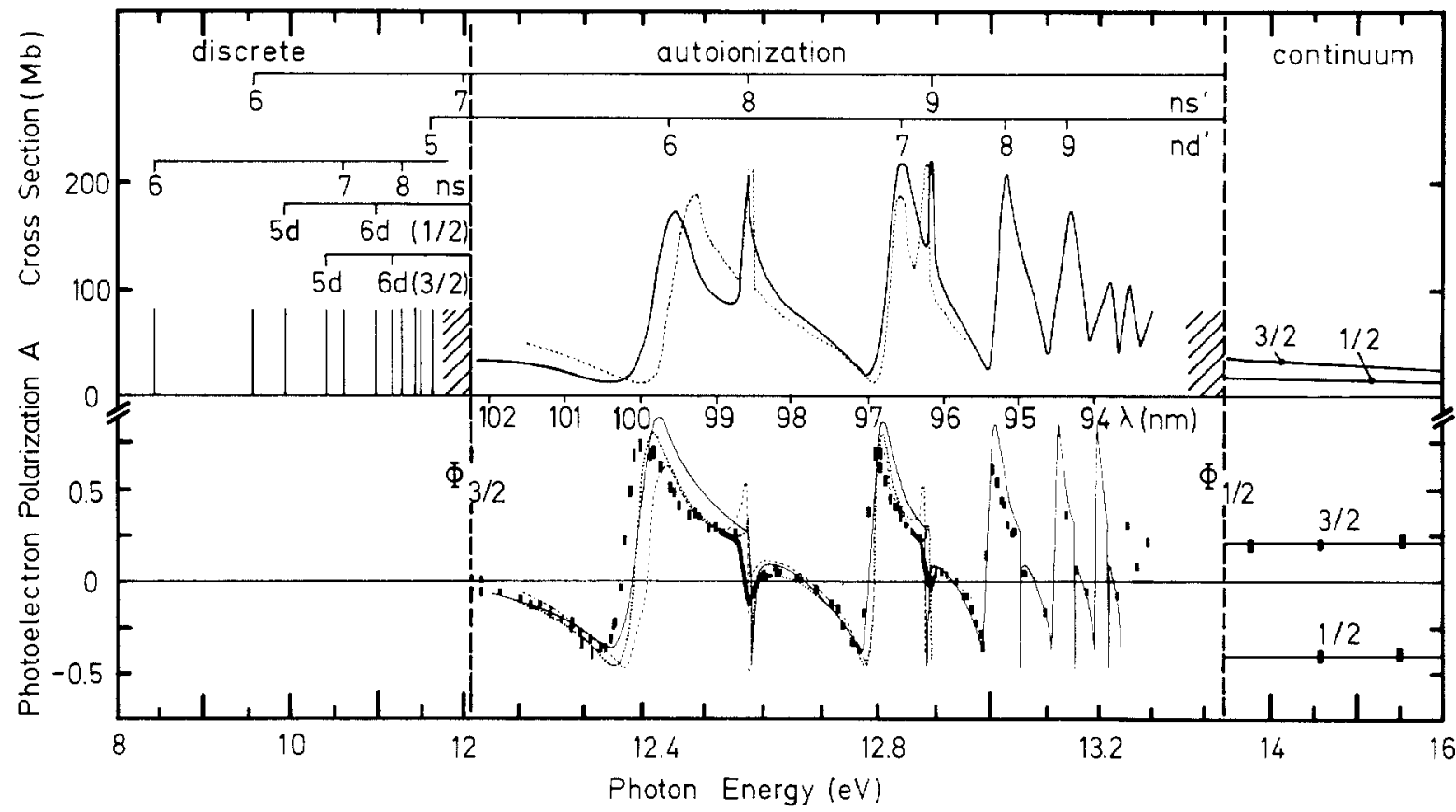

Fig. 9. Photoabsorption of xenon atoms; excitation below the first photoionization threshold $\phi_{3: 2}$ [27] and photoionization above the threshold [9, 23]; upper part: photoionization cross section; lower part: spin parameter

$A$ (which coincides with $A(\theta)$ at $\theta_{\mathrm{m}}=54$, the spin-polarization component parallel to the photon spin, see Fig. 5).

detected via spin-resolved spectroscopy of electrons emitted due to a subsequent Penning-type relaxation mechanism [15]. The spin-polarization results [30] show again the sign sequence as in Fig. 6 for the peaks 1, 2, 3 and provide thus a quantum-number labelling according to Fig. 6 and Fig. 4:

$$
\begin{aligned}
\mathrm{Xe} 5 p^{5}\left({ }^{2} P_{3: 2}\left|M_{j}\right|\right. & =3 / 2) 6 s, \quad 5 p^{5}\left({ }^{2} P_{3 / 2}\left|M_{j}\right|=1 / 2\right) 6 s, \\
5 p^{5}\left({ }^{2} P_{1 / 2}\left|M_{j}\right|\right. & =1 / 2) 6 s
\end{aligned}
$$

which is an electrically neutral complex, where no imagecharge screening for example can occur. This is an evidence that an image-charge mechanism must not be responsible for the $M_{J}$ splitting observed (Fig. 11, Fig. 6). The resonances for the Xe overlayer are closely related to the surface excitons in rare gas crystals known in the literature [32]. This connection is supported by a systematic study of the positions of these resonances as function of the Xe coverage on $\operatorname{Ir}\left(\begin{array}{lll}1 & 1 & 1\end{array}\right)$ up to a three-dimensional crystal [31].

Above the photoemission limits Fig. 11 shows the intensity and the spin-polarization results for normal emission of the three photoelectron peaks $(1,2,3$ as shown in Fig. 6) for a xenon monolayer on $\operatorname{Pt}\left(\begin{array}{lll}1 & 1 & 1\end{array}\right)$ [12] and on graphite [13, 31] as function of the photon energy. Intensity and polarization demonstrate a resonance structure in all three channels, deep minima as well as small enhancements of the photoelectron intensities are correlated with pronounced variations of their spin polarizations. Positions as well as shapes of resonances in channel $2\left({ }^{2} P_{3: 2}\left|M_{J}\right|=1 / 2\right)$ have been found to be independent of the distance between adsorbate and substrate as well as between the adatoms in the adlayer. This can be seen in Fig. 12 in the comparison of this resonance in different adsorbate phases - substrate configurations. 3 monolayer regimes (commensurate $\sqrt{3} \times \sqrt{3}$ Xe layer with a $4.8 \AA$ adatom spacing, incommensurate close packed (hcp) Xe layer with a $4.4 \AA$ adatom spacing, both on Pt( 1111$)$ as well as Xe monolayer on graphite $(0001))[12,13,31]$ are compared with 2 twolayer regimes ( $\mathrm{Xe} / \mathrm{Xe} / \mathrm{Pt}$ and $\mathrm{Xe} / \mathrm{C} / \mathrm{Pt}$ ) [15]. In all

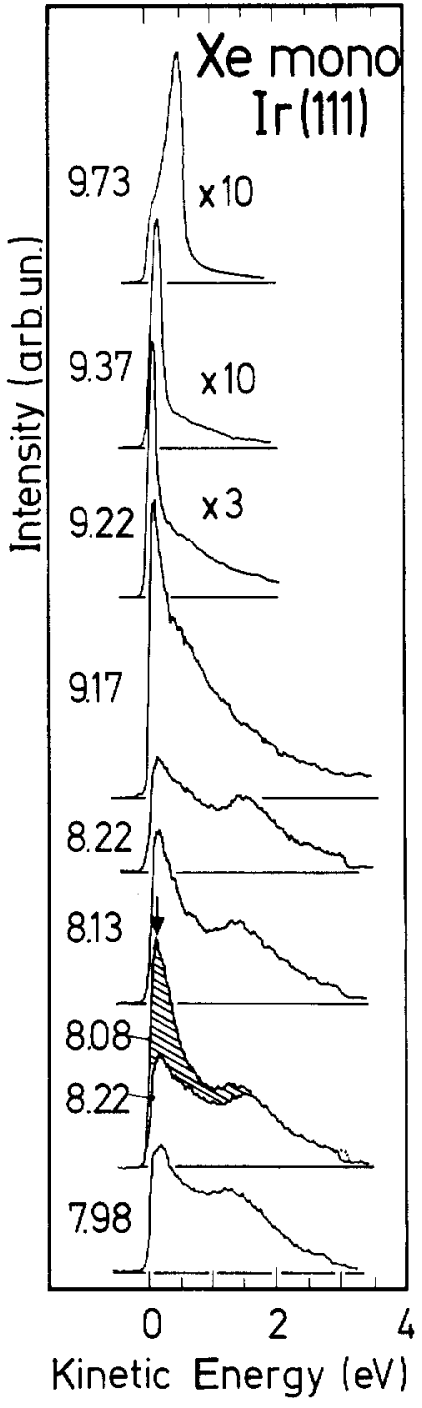

Fig. 10. Electron spectra of Xe monolayer on $\operatorname{Ir}(111)$ with photon energies below the photoemission threshold of a xenon adsorbate [31]. 

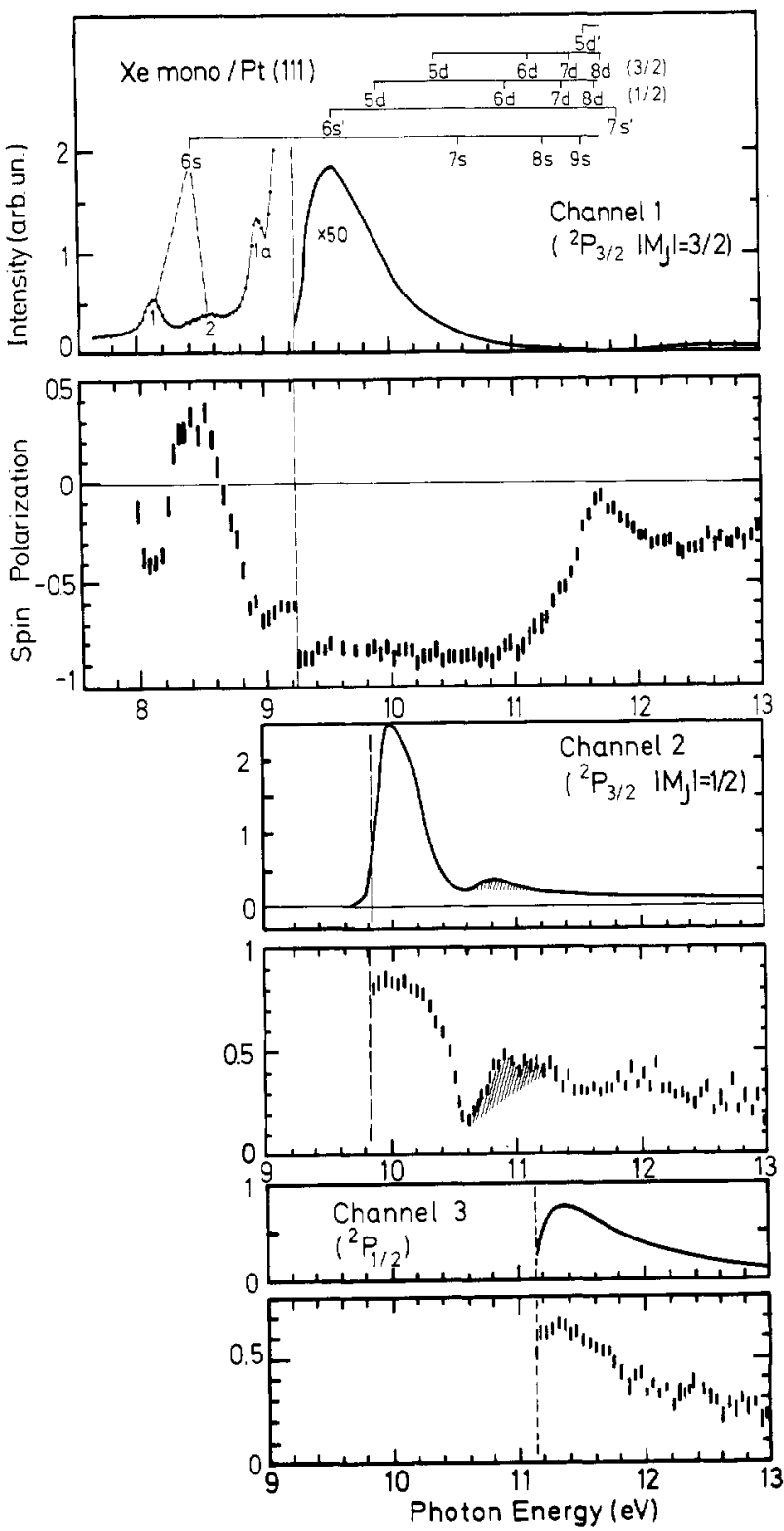

Fig. 11. Spectral dependences of electron intensity and spin polarization for monolayers xenon on $\mathrm{Pt}(111)$ (left) and on graphite $(0001)$ (right). The vertical dashed lines indicate the photoemission thresholds. The channels 1 ,

cases the positions of the resonances (hatched areas) are the same and lie close to the atomic $7 \mathrm{~s}$ excitation (Fig. 9) indicated as arrow in Fig. 12. Because of the insensitivity of this resonance with respect to distances of the xenon atom to the next neighbours the resonance behavior seems to be an atomic Xe like one, may be of the autoionization type due to the position coincidence with the $7 \mathrm{~s}$ atomic level.

That autoionization resonances in photoelectron intensities as well as in spin-polarization components do not only appear in rare-gas systems, but also in open-shell atoms is demonstrated in the next example. Mercury atoms $\left(5 d^{10} 6 s^{2}\right)$ irradiated by vuv radiation of about $20 \mathrm{eV}$ photon energy are photoionized with respect to the $6 s$ as well as to the $5 d$ subshell. The photoelectron spectrum shows a fine structure splitting corresponding to the ionic states ${ }^{2} S_{1 / 2},{ }^{2} D_{5 / 2},{ }^{2} D_{3 / 2}$. Figure 13 shows the positions of both $D$ photoionization thresholds and the spin-parameter values $\alpha$ (describing the height of the spin-polarization component $P_{\mathrm{p}}(\theta)$ in Fig. 5) for both peaks in the photoelectron spectrum corresponding to
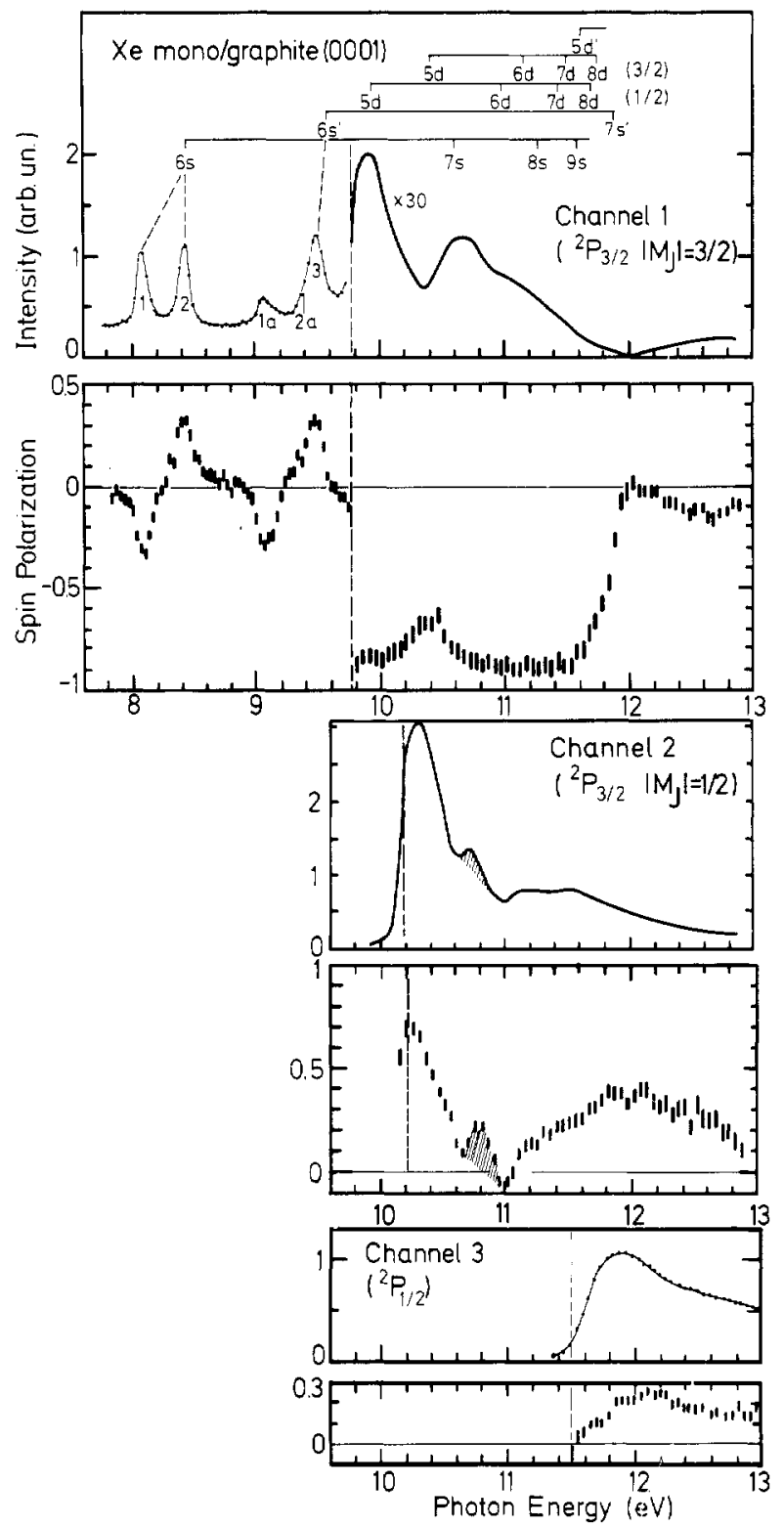

2, 3 refer to the sequence of peaks in Fig. 6 . The positions of atomic resonance lines are from Fig. 9. Experimental data from Refs. [12, 13, 30, 31].

the ionic state configurations ${ }^{2} D_{5 / 2}$ and ${ }^{2} D_{3 / 2}$. The experimental results [33] are in reasonable agreement with a RRPA calculation [34] which includes correlations between 8 channels of $5 d$ and $6 s$ character. Between the two ionization thresholds three Rydberg series converging to the ${ }^{2} D_{3,2}$ threshold couple with continua corresponding with the ${ }^{2} S_{1 / 2}$ and the ${ }^{2} D_{5 / 2}$ ionic states. Figure 14 shows the autoionization resonances measured in the $S_{1 / 2}$ photoelectron peak as example [35]. Photoelectron intensity as well as the spin-polarization components $A(\theta)$ and $P_{\perp}(\theta)$ (Fig. 5) described by the spin parameters $A=$ $A\left(\theta_{\mathrm{m}}\right)$ and $\xi$, respectively, show up a pronounced resonance structure due to the autoionization via the $8 p_{1 / 2}, p_{3 / 2}$ and $5 f_{5 / 2}$ atomic excitation. Further results and details are given elsewhere $[14,35]$.

\section{Symmetry-resolved bandmapping}

The positions of the partial (spin up and down) intensities in the photo-electron spectra described in Section 3 can be used 


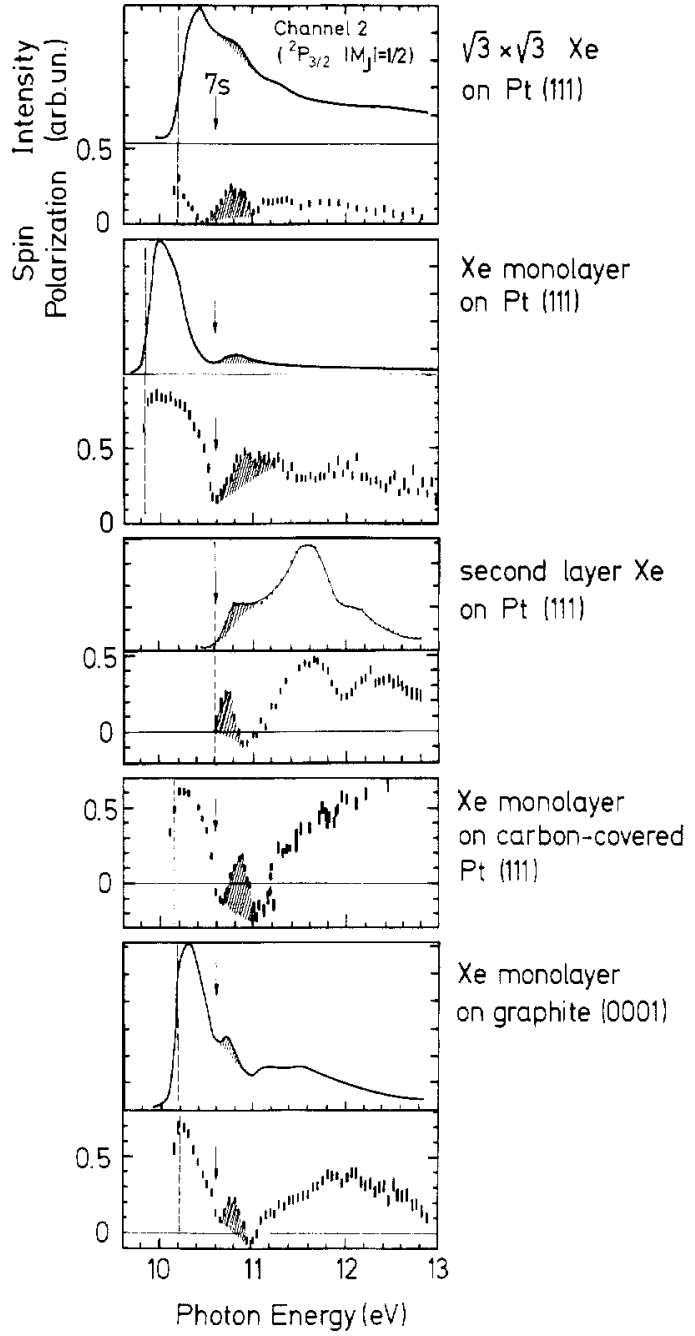

Fig. 12. Spectral dependences of photoelectron intensity and spin polarization in channel 2 (Fig. 11) for photoemission of xenon adsorbates in different phase structures and on different substrates close to the $7 \mathrm{~s}$ excitation energy of a free xenon atom (arrow). Data from Refs. [12, 13, 15, 31].

to perform a band-mapping procedure of the solid state bandstructure spin selected. Every peak gives three pieces of information: (1) the sign of the polarization, (2) the kinetic energy of the electrons, and (3) their binding energy. The photoemission process is discussed in the three step model: direct interband transition, path through the crystal to the surface, escape through the surface into the vacuum. The influence of the last two steps is negligible if the photoemission is studied under normal light incidence and normal

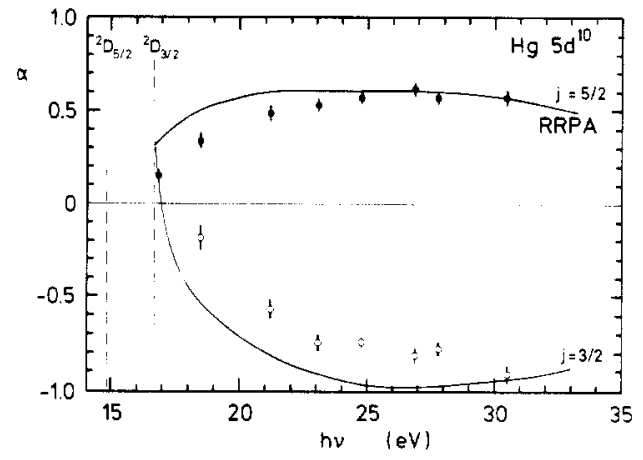

Fig. 13. Energy dependence of the spin parameter $\alpha$ for the $5 d$ photoionization of free mercury atoms, experimental results [33] in comparison with theory [34].

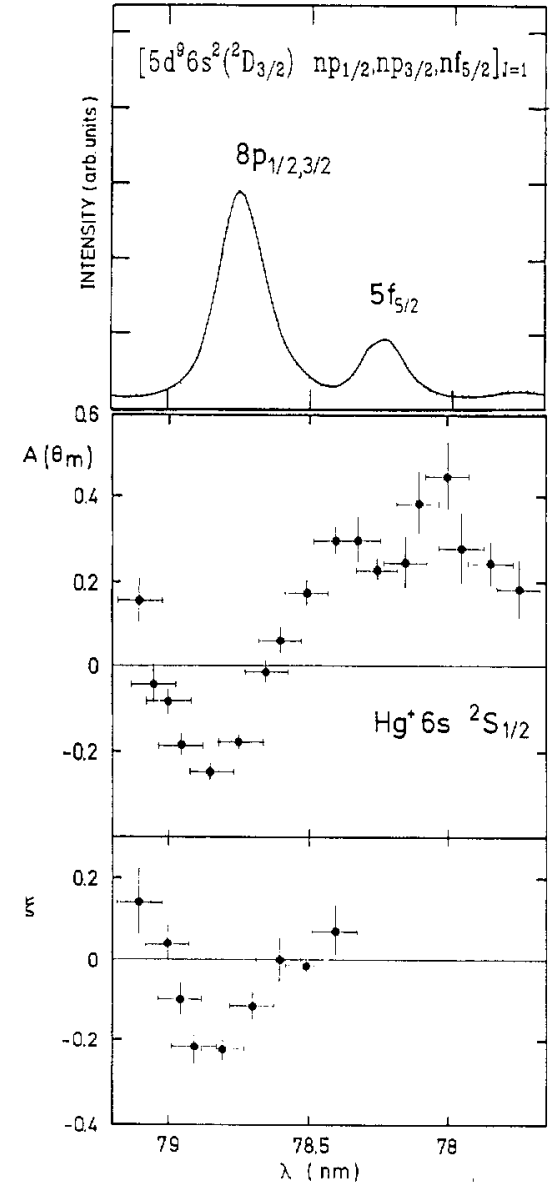

Fig. 14. Photoelectron intensity (upper part) and spin-polarization parameters $A=A\left(\theta_{m}\right)$ and $\xi$ for photoionization of $\mathrm{Hg}$ in the autoionization range; the $\mathrm{Hg}^{+}$ion is in the ${ }^{2} S_{1.2}$ ground state [35]

photoelectron emission. It is self evident that under these conditions a bandmapping, i.e., a determination of initial and final bands by means of the data of the interband transition alone cannot be performed without any knowledge about the behavior of at least one band (the initial or the final one). Therefore the bandmappings discussed here have been done with solid states where bandstructure calculations exist and whose bandstructures are comparable with each other: Pt(l $\left.\begin{array}{lll}1 & 1 & 1\end{array}\right)$ [1 11$]$, $\mathrm{Xe}\left(\begin{array}{lll}1 & 1 & 1\end{array}\right)$ [25] and $\operatorname{Ir}\left(\begin{array}{lll}1 & 1 & 1\end{array}\right)$ [26].

Figure 15 shows a non self-consistent fully relativistic augmented plane wave (RAPW) bandstructure in $\Lambda$ of Pt(l 11 1) [36], which is in excellent agreement with a self consistent bandstructure calculation [37]. Using the spinresolved spectra [11] the valence bands of $\mathrm{Pt}\left(\begin{array}{lll}1 & 1 & 1\end{array}\right)$ below the Fermi level have been mapped starting from the upper calculated band No. 7, which is a free electron parabola up to photon energies of $20 \mathrm{eV}$. The narrowly lying initial bands could be separately mapped by use of the spin information. Thus the theoretically predicted sequence of bands $\Lambda_{4+5}^{3}, \Lambda_{6}^{3}$, $\Lambda_{6}^{1}, \Lambda_{6}^{3}, \Lambda_{4+5}^{3}$ below the Fermi level could be experimentally verified and the bands have been mapped. Since a transition $\Lambda_{6} \rightarrow \Lambda_{6}^{1}$ is forbidden, only 4 direct transitions from the initial states $\Lambda_{4+5}^{3}$ or $\Lambda_{6}^{3}$ did occur yielding negative or positive polarized electrons in the upper band $\Lambda_{6}^{1}$, respectively, as discussed in Section 3.

The band mapping procedure for the insulator $\mathrm{Xe}\left(\begin{array}{lll}1 & 1 & 1\end{array}\right)$ went the opposite way as shown in Fig. 16 [25]. In the occupied region, dispersions were evaluated from the total band width as derived from transitions starting from the 


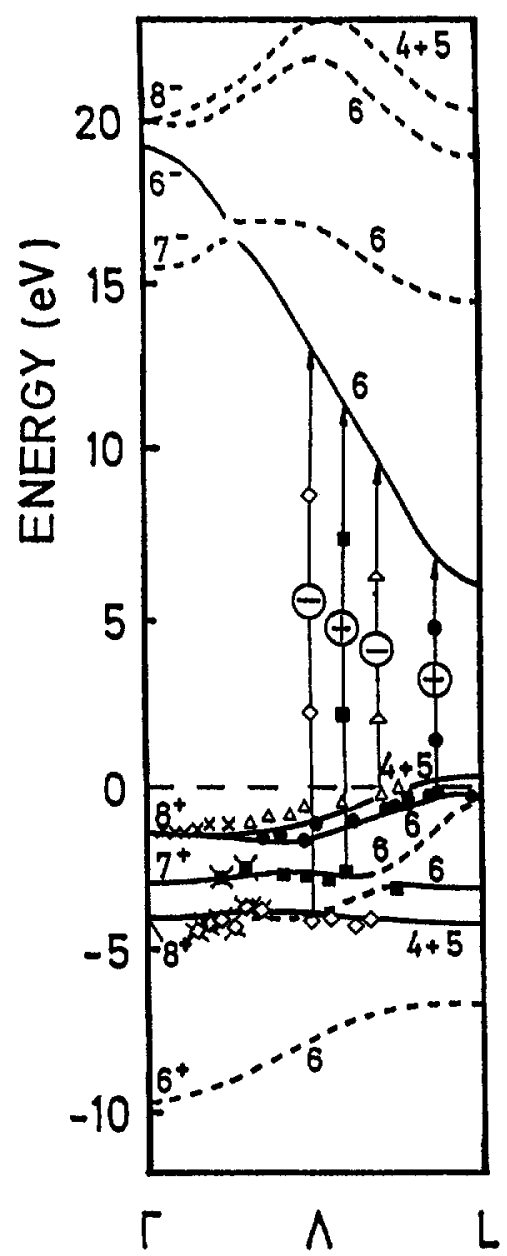

Fig. 15. Symmetry-resolved bandmapping of $\mathrm{Pt}\left(\begin{array}{lll}1 & 1 & 1\end{array}\right)$ in comparison with the calculated bandstructure [11]. The mapping points have been obtained from the spin resolved partial photoelectron spectra. Bands with full symbols yield positive polarized electrons and correspond to $\Lambda_{6}$ symmetry while bands with open symbols (negative polarization) are characterized by $\Lambda_{4+5}$.

occupied bands A, B, C as shown for one photon energy in Fig. 7. Because of the good agreement between this experimental dispersion and the calculation [38] the final bands have been mapped symmetry-resolved starting from the three calculated valence bands slightly modified in their dispersion. The corresponding transitions have been indicated by the symbols $O, \square, \Delta$ in Fig. 16, respectively. They yield positive or negative polarized electrons in the upper final bands according to the selection rules for optical transitions with circularly polarized radiation in $\Lambda$ direction of an f c c crystal [39]. Thus the band mapping procedure demonstrates the $\Lambda_{6}^{1}$ character of the first unoccupied band. It is worth noting that it cannot be interpreted as a free electron parabola, because there is no further branch at higher energies. The higher unoccupied bands shown in Fig. 16 are strongly influenced by hybridization. Thus mapping points of both spin polarization signs occur. These mapping points are in reasonable agreement with a relativistic modification of band-structure calculation of Timmer and Borstel not yet published, as discussed in detail elsewhere [25] and shown in Fig. 16.

For $\operatorname{Ir}\left(\begin{array}{lll}1 & 1\end{array}\right)$ the bandmapping procedures of the initial and of the final states have been combined [26]. The bandstructure of $\operatorname{Ir}\left(\begin{array}{lll}1 & 1 & 1\end{array}\right)$ is similar to that of $\operatorname{Pt}\left(\begin{array}{lll}1 & 1 & 1\end{array}\right)$ as shown in Fig. 17. Besides an experimentally found overall shift of the two upper bands 7 and 8 by $0.8 \mathrm{eV}$ with respect to the cal-

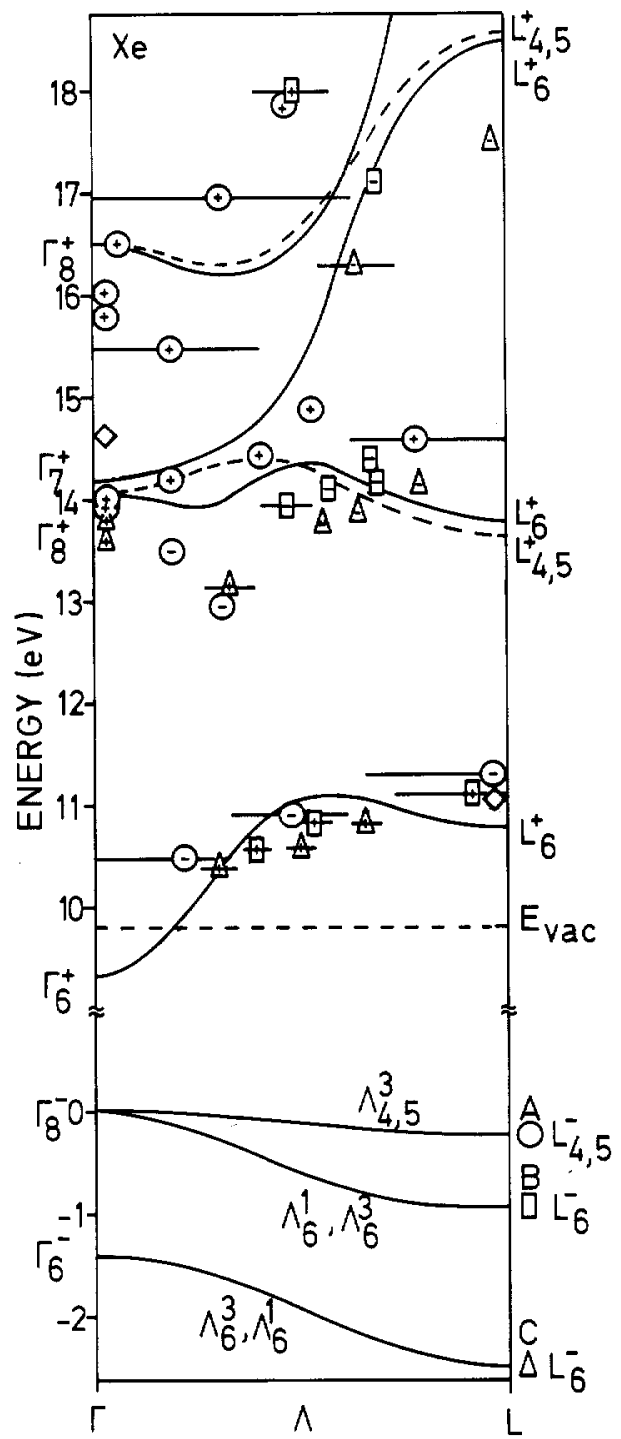

Fig. 16. Experimental band structure [25] of Xe along the $\Gamma-\mathrm{L}$ direction. Circles, squares, and triangles correspond to transitions from the top, second and third occupied band [38], respectively; error bars in the horizontal directions indicate the uncertainty of the mapping procedure in the $k_{\perp}$ direction. Signs indicate the sign of the observed spin polarization.

culated bands [40] the mapping of the 4 valence bands 2,3 , 5,6 by means of the spin resolved partial electron spectra as shown in Fig. 8 demonstrates excellent agreement with theory. Starting from these now experimentally verified initial bands, the small structures in the spin resolved partial spectra in Fig. 8 close to the Fermi energy yields a few mapping points in the second final band No. 8 [26]. It has been characterized with the same symmetry $\Lambda_{6}$ as band No. 7 due to the electron-spin polarization measured. Transitions from the upper-most valence band No. $6\left(\Lambda_{4+5}\right)$ with negative electron polarization in band No. 8 as well as from the valence band No. $5\left(\Lambda_{6}\right)$ with positive polarization in No. 8 could be identified and used for the bandmapping procedure. It is worth to be reminded that without a spin-polarization analysis of the photoelectrons this mapping of band No. 8 could not be performed as shown in Fig. 8. In the total (not spin resolved) intensity there these transitions are only be indicated by a very weak shoulder, whereas the sign switch of the spin polarization lets appear the transitions into the upper 


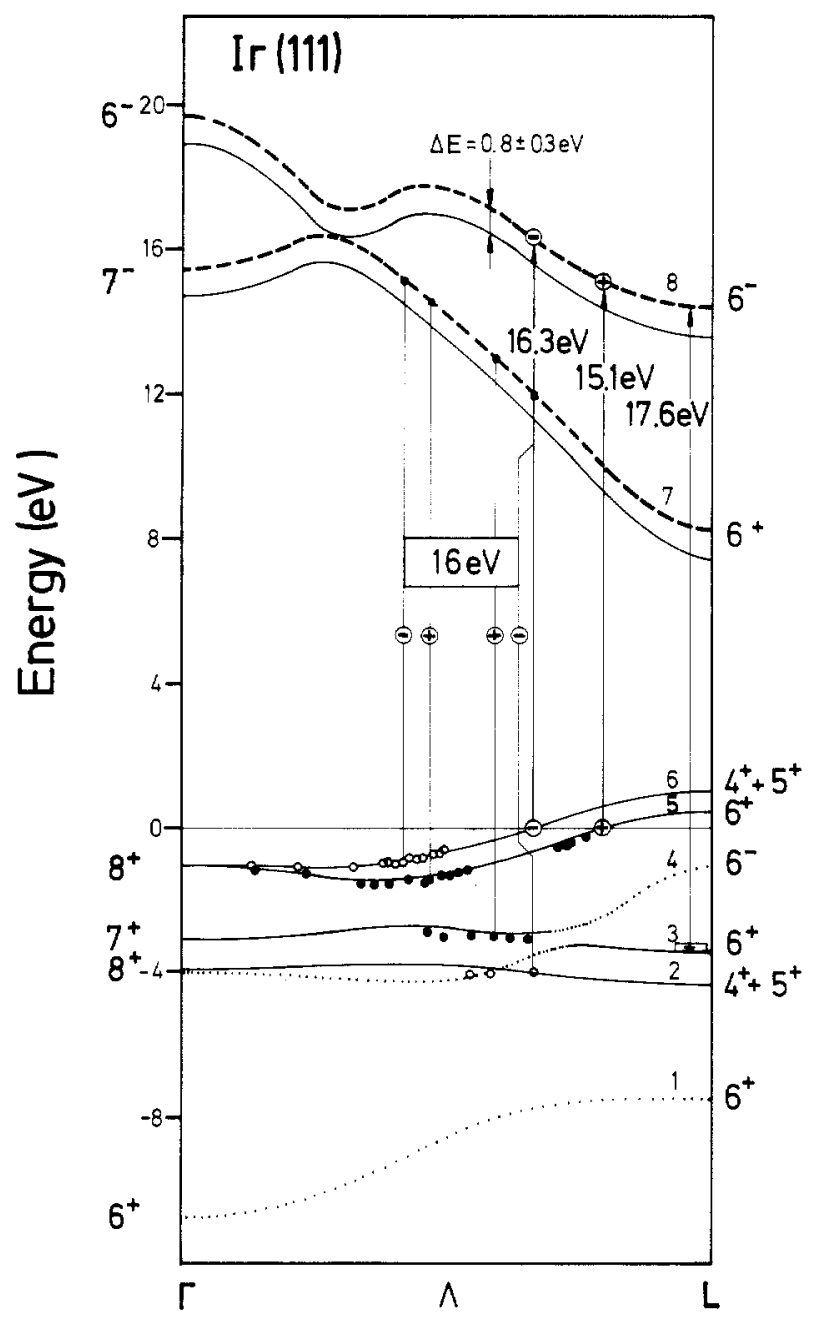

Fig. 17. As Fig. 15 but for $\operatorname{Ir}\left(\begin{array}{lll}1 & 1 & 1\end{array}\right.$ [26]. The calculated bandstructure [40] has to be corrected by a $0.8 \mathrm{eV}$ broader band gap (difference between dashed and full band curves) in order to map the uppermost band between 15.1 and $16.3 \mathrm{eV}$ photon energy well.

band. This demonstrates how spin-polarization spectroscopy may increase the energetic resolution of a photoemission experiment [31].

\section{Complete characterization of the rare-gas photoionization}

As pointed out in Section 3 the photoelectron-emission experiment of xenon atoms has been simultaneously resolved with respect to all variables in the continuum range between $13 \mathrm{eV}$ and $30 \mathrm{eV}$ photon energy as well as in the autoionization region at about $12 \mathrm{eV}$. The experimental information about the total photoionization cross section, the intensity parameter $\beta$, describing the differential cross section, and the 3 spin parameters $A, \alpha$, and $\xi$ describing the behaviors and the angular dependences of the three spin-polarization components $A(\theta), P_{\mathrm{p}}(\theta)$ and $P_{\perp}(\theta)$, respectively, form a complete set of quantum-mechanical parameters.

Figure 9 shows cross section and the spin parameter $A$ in the autoionization range of xenon. Using these as well as the other experimental results for $\beta$ [41] and $\alpha$ and $\xi$ [42] the 5 quantum-mechanical quantities describing the photoionization could be determined [19]: The dipole matrix elements (in atomic units) $D_{1}, D_{2}$, and $D_{3}$ describing transitions into the $d_{5: 2}, d_{3: 2}$ and $s_{1: 2}$ electron continuum state with a remaining $p_{3,2}$ hole $\left(\mathrm{Xe}^{+}\right)$, respectively, as well as the corresponding

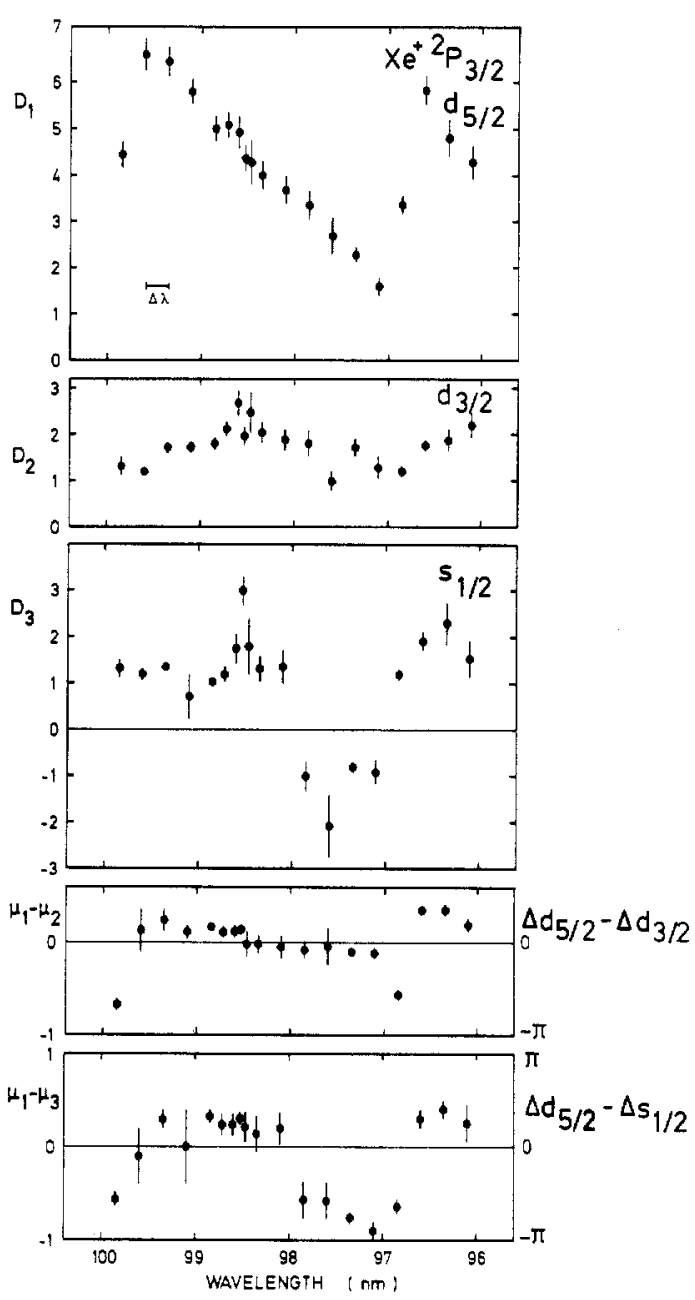

Fig. 18. Experimental matrix elements $D_{i}$ and quantum-defect differences $\mu_{i}$ $-\mu_{i}$ (phase-shift differences) for photoionization of xenon in the $5 p$-autoionization range [19].

phase-shift differences of the continuum wavefunctions $\Delta d_{5: 2}-\Delta d_{3 ; 2}$ and $\Delta d_{5 ; 2}-\Delta s_{1 / 2}$ are shown in Fig. 18 including error bars.

The amplitude $D_{1}$ reflects the wavelength dependence of the photoionization-cross section (upper part of Fig. 9) while the other $d$-matrix element $D_{2}$ does not contribute to the shape of the broad $d$ autoionization resonance. Structures connected with the sharp s-resonances (see Fig. 9) are washed out due to the experimental bandwidth of $0.25 \mathrm{~nm}$ in the spin-polarization experiment. Nevertheless the $s$-resonance at $98.5 \mathrm{~nm}$, however, clearly appears in $D_{3}$ but significantly also in $D_{2}$, the amplitude corresponding to the transition into the $d_{3 / 2}$ continuum, which is evidence of strong interchannel interaction between channels of different orbital angular momenta [9]. Except close to the deep minima of $D_{1}$ and of the total cross section (at $100 \mathrm{~nm}$ and $97 \mathrm{~nm}$ ) the phases of the $d_{5 / 2}$ and $d_{322}$ partial waves are the same, the corresponding phase-shift difference $\mu_{1}-\mu_{2}$ have been found to almost vanish. The phase-shift difference between the $d$ and the $s$ continuum wavefunctions contains additionally to results of Fig. 18 (lowest part) the analytical known Coulomb phase shift difference [19]. For theoretical treatments of the photoionization process by means of the Multichannel quantum defect theory MQDT $[9,28,43,44]$ only the quantum defects $\mu_{1}, \mu_{2}, \mu_{3}$ as shown in Fig. 18 but not the Coulomb phase are of interest. 
Beyond the second photoionization threshold a photoionization into the $d_{3 / 2}$ and $s_{1 / 2}$ continuum channels with a remaining ion in the excited state ${ }^{2} P_{1 / 2}$ (according to a $p_{1 / 2}$ hole in Fig. 4) is possible additional to the channels discussed above. I.e., the photoionization into the continuum region (Fig. 9) is characterized by the dipole matrix elements $D_{i}$ and the corresponding phase shift differences $\delta_{i}$ of the system ion + photoelectron [9] with $J=1$
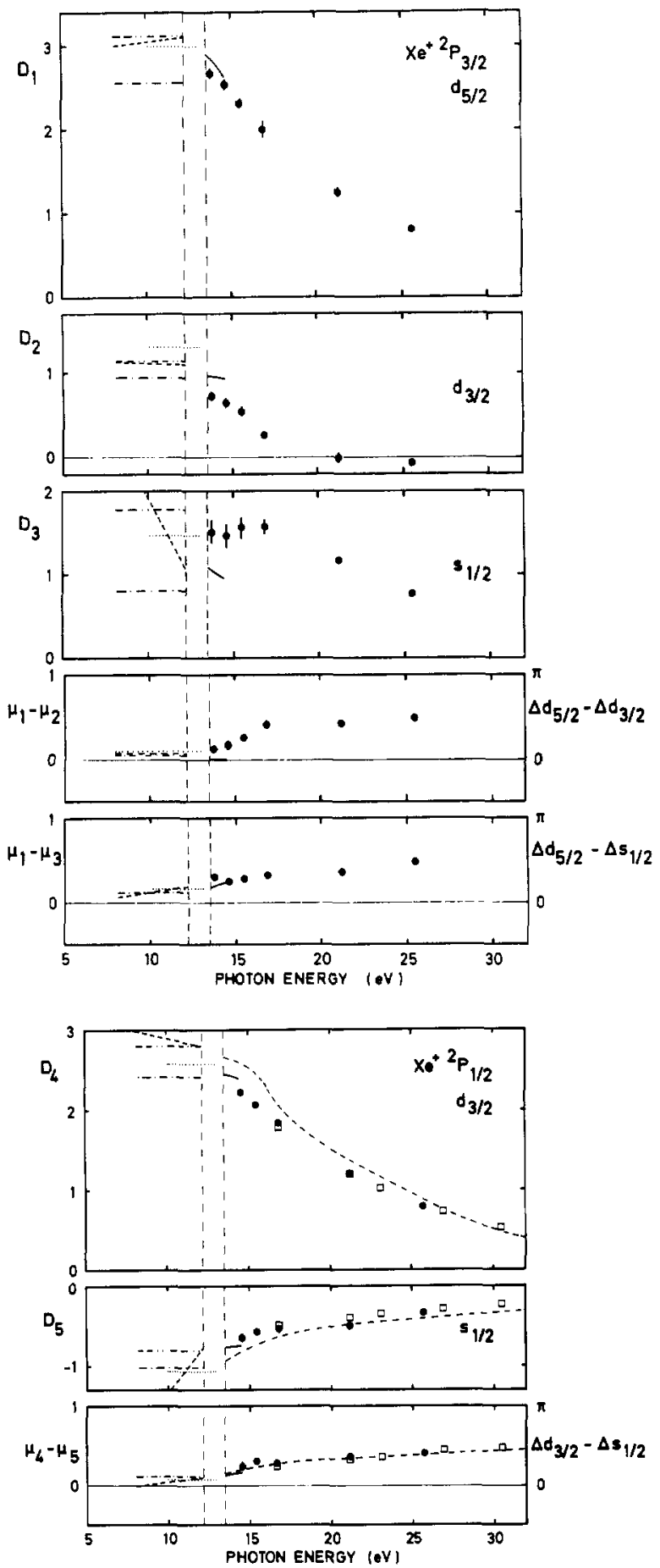

Fig. 19. Matrix elements $D_{i}$ and quantum-defect differences $\mu_{i}-\mu_{j}$ (phaseshift differences) for photoionization of xenon when the ion is in the ${ }^{2} P_{3: 2}$ ground state (a) or in the ${ }^{2} P_{1}$ excited state (b). The experimental points with error bars (full [19], open [9]) are compared with theoretical calculations (dashed [46], full curves [29]) and with data in the discrete spectral range [9] beyond the photoionization thresholds (vertical dashed lines).

$$
\begin{array}{llcc}
i= & 1 & 2 & 3 \\
i= & \left.\begin{array}{ccc}
\left.{ }^{2} P_{3 / 2} d_{5 ; 2}\right)_{1} & \left({ }^{2} P_{3 / 2} d_{3 / 2}\right)_{1} & \left({ }^{2} P_{3 / 2} s_{1 / 2}\right)_{1} \\
& 4 & 5
\end{array}{ }^{2} P_{1 / 2} d_{3 / 2}\right)_{1} & \left({ }^{2} P_{1 / 2} s_{1 / 2}\right)_{1} .
\end{array}
$$

States corresponding to the same final ionic state are energetically degenerate and can interfere with each other. Cross section $Q$, asymmetry parameter $\beta$ as well as the three spin parameter $A, \alpha$ and $\xi$ are known again from different experiments [19] so that an "experimental" determination of all matrix elements $D_{i}$ and all phase-shift differences could be performed again [19]. They are given in Fig. 19(a) and 19(b). Figure 19(a) shows these analogous to Fig. 18 but in the continuum range for the channels with the final ionic state ${ }^{2} P_{3 / 2}$. In application of the MQDT the data shown can be compared with the matrix elements and quantum defects in the discrete spectral range obtained there from values of the oscillator strengths and energy positions of spectral lines in the literature (see Refs. [9, 19]). The cross comparison between photoionization continuum and discrete spectral range across the autoionization region (between the two thresholds in Fig. 19) shows the excellent agreement for the absolute matrix elements and phase-shift differences. It demonstrates the validity of the MQDT and shows that a photoionization threshold is no longer a threshold in the understanding of photoabsorption process of atoms. It is worth noting that in Fig. 19(a) the shape of the photo-ionization cross section is mainly given by the decrease of $D_{1}$ while for $D_{2}$ a Cooper minimum (zero crossing) appears. Furthermore it is interesting that the phase-shift difference between the $d_{5 / 2}$ and the $d_{3 / 2}$ partial waves, which vanishes in the discrete and in the autoionization regions, shows a pronounced energy dependence in the continuum. This experimental result [19] that the phases of two partial waves with equal angular momentum strongly depend upon whether spin- and orbital-angular momentum in the continuum are parallel or antiparallel is a manifestation of a strong spin-orbit interaction in the continuum. The fact that this is connected with the behavior of a Cooper minimum in $D_{2}$ can be explained and further characterized in context of the angular-momentum transfer classification [45] of this photoionization process given elsewhere [19].

Figure 19(b) shows the results for the channels 4 and 5 with the remaining ion in the ${ }^{2} P_{1 / 2}$ state. Additional to the similar results in Fig. 19(a) the experimental data $[9,19]$ are also compared with theoretical calculations $[29,46]$ which show reasonable agreement.

\section{Summary, outlook and acknowledgement}

This review should indicate that the existence of spin-polarized photoelectrons is a common phenomenon in photoionization of atoms and photoemission of adsorbates and nonmagnetic solids rather than exceptional. It is the purpose of the angleand spin-resolved photoelectron spectroscopy to find a set of non-redundant experimental data which characterizes the photoeffect quantummechanically completely. This has been shown for atoms successfully. To build a quantitative bridge from the free atoms, via the free randomly oriented molecules, via the atoms adsorbed and via the free oriented molecules up to the three dimensional crystal will be the main topic of the angle- and spin-resolved photoemission studies in 
the future. Molecular photoionization experiments, spinresolved with randomly oriented molecules [47] and non spin-resolved with free oriented molecules [48] play an important role in this context, but they are not discussed in this paper, because the studies did not use circularly polarized but unpolarized vuv radiation. For rare gas atoms the cross comparison between atomic photoionization and photoemission of adsorbates and insulator crystals could be performed in so far as a joint characterization of the quantum numbers (symmetries) of the states (bands) involved could be performed and as similar resonance processes could be measured and identified. Thus atomic physics can become an applied method to study and to understand more complicated systems like adsorbates and crystals. To extend this method from rare gas atoms to open shell atoms, metals like $\mathrm{Hg}, \mathrm{Pb}, \mathrm{In}$, and to molecules in the gas phase as well as in adsorbates will be the future program of angle-, energy- and spin-resolved photoelectron emission studies using circularly polarized synchrotron radiation.

\section{Acknowledgements}

The author wishes to thank N. Böwering, A. Eyers, Ch. Heckenkamp, B. Kessler. M. Müller. N. Müller, F. Schäfers, B. Schmiedeskamp, and G. Schönhense for their engagement and wealth of ideas in performing the experiments at BESSY and for many intensive discussions. Thanks are due to the colleagues of the Fritz-Haber-Institute and of BESSY for friendly hospitality. Support of BMFT (05331AX) and MPG is gratefully acknowledged.

\section{References}

1. Fano, U., Phys. Rev. 178, 131 (1969).

2. Heinzmann, U., Kessler, J., and Lorenz, J., Phys. Rev. Lett. 25, 1325 (1970).

3. Heinzmann, U., Kessler, J., and Ohnemus, B., Phys. Rev, Lett. 27, 1696 (1971).

4. Pierce, D. T., Meier, F., and Zürcher, P., Phys. Lett. 51A, 465 (1975).

5. Heinzmann, U., Schäfers, F., and Hess, B. A., Chem. Phys. Lett. 69, 284 (1980).

6. Heinzmann, U., Appl. Opt. 19, 4087 (1980)

7. Meier, F. and Pescia, D., in Optical Orientation (Edited by F. Meier and B. Zakharchenya), p. 313ff, North Holland (1984).

8. Kessler, J., Polarized Electrons, 2nd Edition, Springer, Berlin (1985).

9. Heinzmann, U., J. Phys. B13, 4353 and 4367 (1980).

10. Heckenkamp, Ch.. Schäfers, F., Schönhense, G., and Heinzmann, U., Phys. Rev, Lett. 52, 421 (1984).

11. Eyers, A., Schäfers, F., Schönhense, G., Heinzmann, U., Oepen, H. P., Hünlich, K., Kirschner, J., and Borstel. G., Phys. Rev. Lett. 52 , 1559 (1984).

12. Schönhense, G., Eyers, A., Friess, U., Schäfers, F., and Heinzmann. U., Phys. Rev. Lett. 54, 547 (1985).

13. Heinzmann, U. and Schönhense, G., in Polarized Electrons in Surface Physics, (Edited by R. Feder), p. 467ff, World Scientific, Singapore (1985).

14. Heinzmann, U., in Electronic and Atomic Collisions, (Edited by D. C. Lorents, W. E. Meyerhof and J. R. Peterson), p. 37ff, Elsevier Science Publishers B.V., Amsterdam (1986).
15. Schönhense, G., Appl. Phys. A41, 39 (1986).

16. Heinzmann, U., Osterheld, B., and Schäfers, F., Nucl. Instr. Meth. 195, 395 (1982).

17. Schäfers, F., Peatman, W., Eyers, A., Heckenkamp, Ch., Schönhense, G., and Heinzmann, U., Rev. Sci. Instr, 57, 1032 (1986).

18. Heckenkamp, Ch, Eyers, A., Schäfers, F., Schönhense, G., and Heinzmann, U., Nucl. Instr. Meth. A246, 500 (1986).

19. Heckenkamp, Ch., Schäfers, F, Schönhense, G., and Heinzmann. U., Z. Phys. D2, 257 (1986).

20. Huang, K. N., Phys. Rev. A22, 223 (1980)

21. Oepen, H. P., Hünlich, K., Kirschner, J., Eyers, A., Schäfers, F., Schönhense, G., and Heinzmann, U., Phys. Rev. B31, 6445 (1985).

22. Oepen, H. P., Hünlich, K., and Kirschner, J, Phys. Rev. Lett. 56, 496 (1986).

23. Heinzmann, U., in Fundamental Processes in Atomic Collision Physics, (Edited by H. Kleinpoppen, J. S. Briggs, and H. O. Lutz), p. $269 \mathrm{ff}$, Plenum Publ. (1985).

24. Heinzmann, U., Schönhense, G., and Kessler, J., Phys. Rev. Lett. 42. 1603 (1979).

25. Kessler, B., Eyers, A., Horn, K., Müller, N., Schmiedeskamp, B., Schönhense, G., and Heinzmann, U., To be published (1987).

26. Müller, N.. Kessler, B., Schmiedeskamp, B., Schönhense, G., and Heinzmann, U., Sol. State Comm. 61187 (1987).

27. Moore, C. E., Atomic Energy Levels, Vol. III, National Bureau of Standards, U.S.A. (1958)

28. Lee, C. M., Phys. Rev, A10, 1598 (1974).

29. Johnson, W. R., Cheng, K, T.. Huang, K. N., and LeDourneuf, M., Phys. Rev. A22, 989 (1980).

30. Schönhense, G., Eyers, A., and Heinzmann, U., Phys. Rev. Lett. 56, 512 (1986).

31. Schönhense, G., Kessler, B., Schmiedeskamp, B., Müller, N., and Heinzmann, U., VUV 8, Lund, Sweden. 1986, book of abstracts p. 518 and Proceedings, Physica Scripta, Vol. 35 (1987).

32. Saile, V., Skibowski, M., Steinmann. W., Gürtler, P., Koch, E. E., and Kozevnikov, A., Phys. Rev. Lett. 37305 (1976).

33. Schäfers, F., Heckenkamp, Ch., Schönhense, G., and Heinzmann, U., 2. ECAMP, Amsterdam, 1985, book of abstracts p. 80.

34. Johnson, W. R., Radojevic, V., Deshmukh, P., and Cheng, K. T., Phys. Rev. A25, 337 (1982).

35. Schäfers, F., Müller, M., Böwering, N., Heckenkamp, Ch., and Heinzmann, U., VUV 8, Lund, Sweden, 1986, book of abstracts p. 508 and Proceedings, Physica Scripta, Vol. 35 (1987).

36. Andersen, O, K., Phys. Rev. B2, 883 (1970).

37. Leschik, G.. Courths, R., Wern, H., Hüfner, S., Eckhardt, H., and Noffke, J., Solid State Comm. 52, 221 (1984).

38. Horn, K., Bradshaw, A. M., Noffke, J., and Hermann, K., VUV 6, Charlottesville, 1980 , book of abstracts I 32 .

39. Wöhlecke, M. and Borstel, G., p. 423 in Ref. [7].

40. Noffke, J. and Fritsche, L., J. Phys. F: Met. Phys. 12, 921 (1982)

41. Samson, J. A. R. and Gardner, J. L., Phys. Rev. Lett. 22, 1327 (1973).

42. Heckenkamp, Ch., Schäfers, F., Schönhense, G., and Heinzmann, U., Phys. Rev. A32, 1252 (1985).

43. Seaton, M. J., Comm. At. Mol. Phys. 2, 37 (1970)

44. Fano, U., J. Opt. Soc. Am. 65, 979 (1975).

45. Dill, D., Phys. Rev. A7, 1976 (1973).

46. Amusia, M. Ya. and Ivanov, V. K., Izv. Akad. Nauk SSSR, Ser. Fiz 41, 2509 (1977).

47. Schönhense, G., Dzidzonou, V., Kaesdorf, S, and Heinzmann, U., Phys. Rev. Lett. 52, 811 (1984)

48. Kaesdorf, S., Schönhense, G, and Heinzmann, U., Phys. Rev. Lett. 54. 885 (1985). 